\title{
Clinical Diagnosis of Metabolic Syndrome 4. Therapy for Metabolic Syndrome
}

\author{
Koichi Node \\ Key words: insulin resistance, atherosclerosis, vascular endothelial function
}

(DOI: 10.2169/internalmedicine.46.1906)

There are several cardiovascular risk factors that accompany the metabolic syndrome: dyslipidemia, elevated blood pressure, elevated glucose and a pro-inflammatory state. The likelihood of an individual developing metabolic syndrome is enhanced by the underlying risk factors: obesity, insulin resistance, lack of physical activity and age. In addition to being at higher risk for atherosclerotic cardiovascular disease, persons with the metabolic syndrome are at increased risk for type 2 diabetes. Patients with the metabolic syndrome deserve management in the clinical setting to reduce the risk for both atherosclerosis and type 2 diabetes. The two major therapeutic strategies for treatment of affected persons are modification of the underlying risk factors and separate drug treatment for the particular metabolic risk factors. Therapy for underlying risk factors is therapeutic lifestyle changes, such as weight loss, increased physical activity. Whether use of drugs to reduce insulin resistance is effective, safe, and cost-effective before the onset of diabetes awaits the results of more clinical research in addition to
PROACtive study and UKPDS23. Turning to individual risk components, for dyslipidemia, drug therapies that promote lowering of triglyceride and raise HDL cholesterol will be needed for higher risk patients. Fibrate might be the first choice for metabolic syndrome. Treatment of categorical hypertension including masked hypertension with drugs has become standard practice. When hyperglycemia reaches the diabetic level, glucose-lowering agents will become necessary, and reduction of a prothrombotic state with aspirin may be indicated in higher-risk patients. In terms of reducing the insulin resistance, angiotensin receptor antagonist, angiotensin-converting enzyme inhibitor, HMG-CoA reductase inhibitor (statin) might be useful for patients with metabolic syndrome. Vascular endothelial function is another target for treatment of insulin resistance, because endothelial dysfunction is correlated with insulin resistance. Prospective randomized trials for metabolic syndrome are necessary to establish the appropriate therapy.

\footnotetext{
(C) 2007 The Japanese Society of Internal Medicine http://www.naika.or.jp/imindex.html
} 